\title{
Corrigendum: Beyond Stereotypes: Analyzing Gender and Cultural Differences in Nonverbal Rapport
}

\author{
Gary Bente ${ }^{1}$, Eric Novotny ${ }^{2 *}$, Daniel Roth ${ }^{3}$ and Ahmad Al-Issa ${ }^{4}$ \\ ${ }^{1}$ Department of Communication, Michigan State University, East Lansing, MI, United States, ${ }^{2}$ Grady College of Journalism \\ and Mass Communication, University of Georgia, Athens, GA, United States, ${ }^{3}$ Department of Computer Aided Medical \\ Procedures and Augmented Reality, Technical University of Munich, Munich, Germany, ${ }^{4}$ Department of English, American \\ University of Sharjah, Sharjah, United Arab Emirates
}

Keywords: rapport, nonverbal behavior, motion capture, character animation, gender, culture

\section{A Corrigendum on}

Beyond Stereotypes: Analyzing Gender and Cultural Differences in Nonverbal Rapport by Bente, G., Novotny, E., Roth, D., and Al-Issa, A. (2020). Front. Psychol. 11:599703. doi: $10.3389 /$ fpsyg.2020.599703

In the original article, there was an error.

In section Method: Behavior Analysis, Parameter Formation, Paragraph 4, we said: "From DTW we calculated the length of the optimal warp path as a measure of similarity of the behavior vectors when stretched or compressed in time." This is incorrect. We did not use "path length" but "DTW distance" for further statistical analyses.

A correction has been made to the section Method: Behavior Analysis, Parameter Formation, Paragraph 4. The corrected paragraph is shown below.

Pearson " $r$ " and the entropy measure resulting from MI analysis were used as input for statistical analysis. From RWTLCC we calculated the average maximal correlation at each point across all time lags as well the absolute offset of the correlation peak from the zero lag as general coherence and synchrony indicators. We further used the "DTW distance" measure, i.e., the minimum path cost (Cheong, 2019), to quantify the (dis)similarity between the behavioral vectors of the interactants for further analyses.

Additionally, there was also an error in Method: Observer Study, Stimulus Material, Paragraph 2. In the phrase "Cameras were around a square area of $4 \times 4$ meters," the word "positioned" is missing.

A correction has been made to the section Method: Observer Study, Stimulus Material,

This article was submitted to Personality and Social Psychology, a section of the journal Frontiers in Psychology

Received: 02 March 2021 Accepted: 03 March 2021 Published: 15 April 2021

Participants were instructed that they would have a short 5-7 min conversation with another student during which they should get to know each other. Before the conversations began, participants were led into different rooms to put on the datasuits necessary for motion capturing. A same sex student assistant placed the markers on the data suits and guided the interactors to the middle of the recording room where they met the experimenter. Motion capture was performed with a 12-camera Optitrack system and the capture software Arena (Optitrack, 2017). Cameras were positioned around a square area of $4 \times 4$ meters. Participants were then asked by the experimenter to take a T-pose (upright symmetric posture with legs closed and arms horizontally stretched out, palms down) for calibration of the tracking system. Then the participants were told that they could move freely in the square between the cameras and should use the next 5-7 min to get to know each other. Next, the experimenter left the room and the participants started the 
conversation. Using the capture software Arena, full body motion of both actors was captured during the conversation with a temporal resolution of $150 \mathrm{~Hz}$. Figure 1 shows a dyad wearing the data-suits with the IR reflecting markers and a projection of the capture software showing both virtual characters for demonstration purposes. After completing the interaction, the

\section{REFERENCES}

Cheong, J. H. (2019). Four Ways to Quantify Synchrony Between Time Series Data. Towards Data Science. Available online at: https://towardsdatascience.com/ four-ways- to-quantify-synchrony-between-time-series-data-b99136c4a9c9 (accessed March 2020).

Optitrack (2017). Optitrack [Motion Capture Hardware]. Available online at: http:// optitrack.com (accessed 2017). participants were debriefed and received 15 Euro (Cologne), or an equivalent on-campus restaurant voucher (Sharjah) for their participation.

The authors apologize for these errors and state that they do not change the scientific conclusions of the article in any way. The original article has been updated.

Copyright (c) 2021 Bente, Novotny, Roth and Al-Issa. This is an open-access article distributed under the terms of the Creative Commons Attribution License (CC BY). The use, distribution or reproduction in other forums is permitted, provided the original author(s) and the copyright owner(s) are credited and that the original publication in this journal is cited, in accordance with accepted academic practice. No use, distribution or reproduction is permitted which does not comply with these terms. 